\title{
Double-Pass Measurement of Retinal Image Quality in the Chicken Eye
}

\author{
NANCY J. COLETTA, OD, PhD, FAAO, SUSANA MARCOS, PhD, \\ CHRISTINE WILDSOET, BScOptom, PhD, and DAVID TROILO, PhD \\ New England College of Optometry, Boston, Massachusetts (NJC, DT), Instituto de Optica (CSIC), Madrid, Spain (SM), School of \\ Optometry, University of California, Berkeley, California (CW)
}

\begin{abstract}
Purpose. The chicken, Gallus gallus domesticus, is used as an animal model to study the development of refractive error. Although vision is important in determining the eye's refractive state, relatively little is known about the retinal image quality of the chicken eye. An objective double-pass technique was used to measure the optical quality of the eyes of White Leghorn chickens. Methods. Measurements were made on 21 eyes of six untreated birds and eight experimental birds that were members of a study of refractive development. Ages ranged from 3 to 6 weeks, and refractions ranged from -1.29 to $+0.58 \mathrm{D}$ in the untreated eyes and -4.58 to $+10.17 \mathrm{D}$ in the experimental eyes. The measurements were made under general anesthesia combined with either cycloplegia or ciliary nerve section. Proper optical alignment of the eye was achieved with the aid of a TV monitor, CCD camera, and an infrared source. A 543-nm laser point source was focused on the retina, and the double-pass aerial image was collected by a high-resolution CCD camera. Refractive errors were corrected with trial lenses, using a bracketing method to optimize the retinal images. Both the full width at half-maximum of the double-pass aerial image and the single-pass modulation transfer function were used as objective estimates of the optical quality. Results. The mean full width at half-maximum value in eyes of the untreated birds was $1.60 \mathrm{~min}$ arc for a $4.50-\mathrm{mm}$ mean pupil diameter. Optical quality tended to be worse in the experimental myopic eyes. Conclusions. The optical quality of the chicken eye measured under monochromatic conditions meets or may even exceed the neural limits of spatial acuity based on anatomical estimates of ganglion cell spacing. The data also suggest that optical quality is worse in myopic eyes, which is consistent with studies of human eyes. (Optom Vis Sci 2003;80:50-57)
\end{abstract}

Key Words: chicken, optical quality, aberrations, modulation transfer function, refractive error, myopia, animal model

$\mathrm{n}$ the field of myopia research, interest in the optical aberrations of the eye and their influence on the quality of the retinal image has gained momentum because of their implications for optimizing refractive surgery techniques. However, there are also now a number of reports linking myopia with increased monochromatic aberrations, ${ }^{1-3}$ raising the possibility that these aberrations may be causal. It has been shown for both the chicken and monkey in experimental studies of eye growth regulation that even relatively subtle retinal image degradation can cause myopic growth., 5

The chicken is the most commonly used animal model for studies of emmetropization and myopia. However, although basic optical models of the chicken eye have been developed, ${ }^{6,7}$ there are no published studies of the optical aberrations of the chicken eye. Because ocular aberrations directly impact the quality of the retinal image that, in turn, impacts on the effectiveness of emmetropization, a specific study of retinal image quality is overdue. The aber- rations of the eye are also of interest as a potential source of information about defocus that might be used to decode its sign during emmetropization. ${ }^{8,9}$

In this study, we present an initial investigation of the optical quality of the chicken eye using a rapid objective double-pass technique that has already been applied to the study of mammalian animal models, such as the cat ${ }^{10}$ and rat. ${ }^{11}$ It has also been used extensively to study the optical quality of the human eye. ${ }^{12,13}$

\section{METHODS}

Measurements were made on 21 eyes of 14 White Leghorn chickens (Gallus gallus domesticus). Ten of these eyes were from six untreated birds, and the other 11 eyes were from eight birds that were members of a separate study of experimental emmetropization (summarized in Table 1). Of the six untreated birds, measure- 
ments were made on one eye of each of two 5-week old birds and both eyes of four 6 -week old birds. The refractive errors of these untreated eyes ranged from -1.29 to $+0.58 \mathrm{D}$ based on the average focus position of the three narrowest double-pass images for each eye. Data represent spherical equivalent values. Typically, chickens are slightly hyperopic at this age, ${ }^{6}$ although the two 5 -week-old birds in this study were both slightly myopic.

The eight experimental birds were 3 to 4 weeks of age. Of these eight birds, five underwent monocular ciliary nerve section (CNS) shortly after hatching; four of these eyes were fitted with monocular spherical lenses 2 weeks later (lens + CNS). ${ }^{14}$ The refractive errors of the lens-treated eyes of these CNS birds ranged from -3.42 to $+10.17 \mathrm{D}$. Their fellow eyes were not measured. The remaining three experimental birds (lens only) were fitted with monocular lenses at 2 weeks of age. The refractive errors of the lens-treated eyes of this group ranged from -4.58 to $+10.00 \mathrm{D}$, and those of their fellow control eyes ranged from plano to $+0.67 \mathrm{D}$.

Internal axial lengths, from the anterior cornea to the internal limiting membrane of the retina, were available for all eyes except the two 5-week old untreated eyes. These data were obtained using high frequency A-scan ultrasonography under halothane anesthesia $^{15}$ and are listed in Table 1 . Axial length varied with age, increasing by $0.59 \mathrm{~mm} /$ week (linear regression analysis: axial length in $\mathrm{mm}=7.74+0.59 \times$ age in weeks; $\left.\mathrm{R}^{2}=0.83, \mathrm{p}<0.0001\right)$, which is consistent with published data for normal chickens. ${ }^{6}$ As expected, myopic eyes were longer than hyperopic eyes in eyes of the same age. In the current study, age contributed more to the variability in axial length than refractive error.

\section{Optical System}

The optical quality of the chick eyes was assessed using a doublepass technique. Fig. 1 illustrates the theory behind this technique. Light from a point source $(\mathrm{O})$ is brought to a focus on the retina. The retinal image of the point source is the eye's point-spread function (PSF), indicated as $\mathrm{O}^{\prime}$. Some of the light from the retinal image, probably originating from the photoreceptor inner segment layer, reflects back out of the eye. The reflected light forms an aerial image $\left(\mathrm{O}^{\prime \prime}\right)$ that is conjugate with the retina. The light forming the aerial image has passed through the eye's optics twice and hence is called the double-pass aerial image. The double-pass aerial image represents the autocorrelation of the single-pass retinal $\mathrm{PSF}^{16}$ and can be analyzed to estimate the optical quality of the eye. The square root of the Fourier transform of the double-pass aerial image provides the single-pass modulation transfer function (MTF). This function is typically shown graphically as the ratio of the retinal image contrast to object contrast, plotted as a function of spatial frequency.

For the current study, the source for the double-pass system was a 543-nm helium-neon laser. The laser beam passed through a spatial filter with a $40 \times$ microscope objective that focused the

\section{TABLE 1.}

Summary of chickens used in this study ${ }^{a}$

\begin{tabular}{|c|c|c|c|c|c|c|c|c|}
\hline Bird & Eye & Status & $\begin{array}{l}\text { Age } \\
(w k)\end{array}$ & $\begin{array}{l}\text { Axial Length } \\
\quad(\mathrm{mm})\end{array}$ & $\begin{array}{l}\text { Lens Power } \\
\text { Worn (D) }\end{array}$ & $\begin{array}{l}\text { Lens Wear } \\
\text { Duration (wk) }\end{array}$ & $\begin{array}{l}\text { Refraction } \\
\text { (D) }\end{array}$ & $\begin{array}{l}\text { Pupil Diameter } \\
\qquad(\mathrm{mm})\end{array}$ \\
\hline A & OD & Untreated & 5 & $\mathrm{n} / \mathrm{a}$ & - & - & -1.00 & 3.00 \\
\hline B & OD & Untreated & 5 & $\mathrm{n} / \mathrm{a}$ & - & - & -1.29 & 3.50 \\
\hline C & OD & Untreated & 6 & 11.189 & - & - & +0.58 & 5.50 \\
\hline $\mathrm{C}$ & OS & Untreated & 6 & 11.147 & - & - & +0.25 & 5.00 \\
\hline D & OD & Untreated & 6 & 11.264 & - & - & +0.25 & 5.25 \\
\hline D & OS & Untreated & 6 & 11.192 & - & - & -0.25 & 5.25 \\
\hline E & OD & Untreated & 6 & 10.908 & - & - & +0.08 & 3.50 \\
\hline E & OS & Untreated & 6 & 10.924 & - & - & 0.00 & 4.00 \\
\hline $\mathrm{F}$ & OD & Untreated & 6 & 11.657 & - & - & +0.33 & 5.00 \\
\hline $\mathrm{F}$ & OS & Untreated & 6 & 11.580 & - & - & -0.50 & 5.00 \\
\hline G & OD & Lens only & 3 & 9.440 & +5.00 & 1 & +4.58 & 3.00 \\
\hline G & OS & Fellow control eye & 3 & 9.617 & - & - & +0.67 & 3.00 \\
\hline $\mathrm{H}$ & OD & Lens only & 3 & 9.773 & -5.00 & 1 & -4.58 & 3.00 \\
\hline $\mathrm{H}$ & OS & Fellow control eye & 3 & 9.426 & - & - & 0.00 & 2.50 \\
\hline I & OD & Lens only & 3 & 8.964 & +10.00 & 1 & +10.00 & 2.00 \\
\hline I & OS & Fellow control eye & 3 & 9.544 & - & - & +0.17 & 2.00 \\
\hline J & OD & $\mathrm{CNS}$ & 3 & 9.163 & - & - & -0.67 & 3.80 \\
\hline K & OD & Lens + CNS & 3 & 9.120 & -5.00 & 1 & -3.42 & 4.00 \\
\hline L & OD & Lens + CNS & 4 & 10.841 & +5.00 & 2 & +4.92 & 4.75 \\
\hline M & OD & Lens + CNS & 4 & 11.050 & -10.00 & 2 & -2.87 & 4.25 \\
\hline $\mathrm{N}$ & OD & Lens + CNS & 4 & 10.134 & +10.00 & 2 & +10.17 & 4.00 \\
\hline
\end{tabular}

${ }^{a}$ Birds labeled A-F were untreated. Birds G-I wore monocular lenses starting at age 2 weeks, whereas their fellow control eye was untreated; their lens-treated eyes are referred to as "lens-only." Birds J-N underwent ciliary nerve section (CNS) shortly after hatching; four of these $\left(\mathrm{K}-\mathrm{N}\right.$, referred to as "lens $\left.+\mathrm{CNS}^{\prime}\right)$ also wore monocular lenses starting at age 2 weeks. Ultrasound measurements were unavailable for birds A and B. Refractions were determined as the average of the lens powers that resulted in the three best double-pass aerial images. Pupil diameters were measured during the double-pass measurements. 


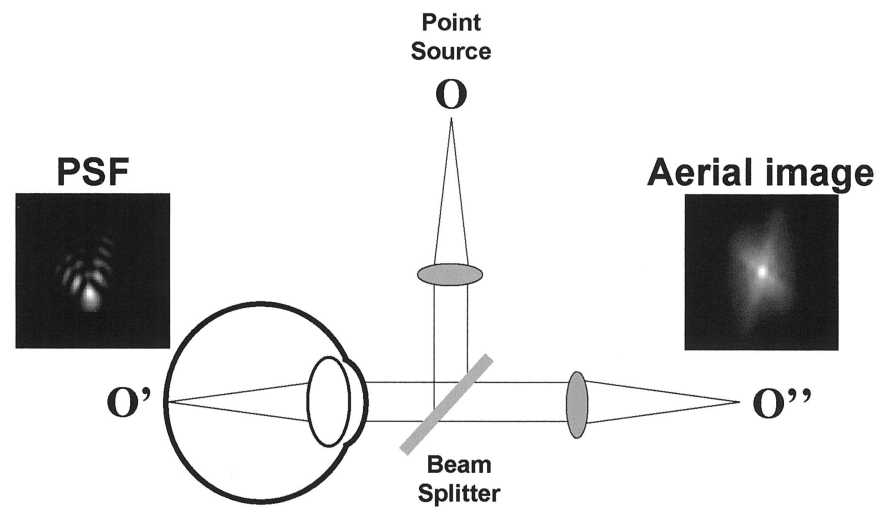

FIGURE 1.

Theory of the double-pass technique. See text for details. O, point source; $\mathrm{O}^{\prime}$, retinal image of the point source (shown left inset as point spread function [PSF]); $\mathrm{O}^{\prime \prime}$, image formed by light reflected from the retina (shown in right inset as aerial image).

beam onto a $5-\mu \mathrm{m}$-diameter pinhole that served as a point source. After diverging from the pinhole, the beam was collimated and passed through a 5-mm-diameter aperture. It then entered the eye after reflection at a beam-splitter cube and formed the retinal PSF $\left(\mathrm{O}^{\prime}\right)$. The reflected beam from the retina was collected by a 500-mm focal-length lens that focused the double-pass aerial image $\left(\mathrm{O}^{\prime \prime}\right)$ at a scientific-grade cooled CCD (Princeton Instruments). The optical system was calibrated using an artificial eye that consisted of a diffraction-limited 50 - $\mathrm{mm}$ focal-length positive lens and a black cardboard "retina" in the lens focal plane. The Strehl ratio of the system was in the range of 0.8 to 0.9 with the artificial eye in best focus, indicating that the system was diffraction-limited.

\section{Procedures}

The experimental procedures were conducted in accordance with the National Institutes of Health Guide for the Care and Use of Laboratory Animals, and the study was approved by the Animal Care and Use Committee of the New England College of Optometry. For measurement, chickens were anesthetized with chloropent $(0.3 \mathrm{ml}$ per $100 \mathrm{~g}$ body weight). The eyes of birds that had not undergone ciliary nerve section were treated with topical vecuronium bromide ( 5 drops of $1 \mathrm{mg} / \mathrm{ml}$ solution at 5-min intervals) to dilate their pupils and paralyze accommodation. During recording, birds rested on a positioning stage with a head restraint that could be adjusted to align their eyes with the optical system. A lid retractor held the eyelid open, but left the eye's nictitating membrane free to move during measurements. This strategy allowed regular renewal of the tear layer and so largely removed tear film breakdown as a source of image degradation in the images recorded. To obtain optimal ocular alignment, eyes were illuminated with an infrared source and viewed with a CCD video camera attached to a monitor. With the aid of this imaging system, the pupil of the eye was centered in the optical system; the Purkinje image of this infrared source was also used in aligning the optical axis of the eye to that of the optical system. Pupil diameter was measured by comparing the video image of the pupil with a calibrated reti- cule transparency mounted on the video monitor. Once alignment was achieved, the infrared source was turned off, and recording commenced.

Images were recorded using a camera exposure duration of $1 \mathrm{~s}$. Corrected-curve trial lenses were added sequentially, just in front of the eye to obtain the best focus (narrowest aerial image) of the point source on the retina. Both spherical and cylindrical lenses, available in $0.12 \mathrm{D}$ steps, were used as needed. A series of doublepass images was captured encompassing various focus states for each eye, although a systematic "through-focus" study of optical quality was attempted in only two birds.

Overexposure of the retina to the laser used to generate the retinal point source was avoided by blocking its path between recordings; eye alignment was rechecked at the same time. A 0.5$\log$ unit neutral-density filter attenuated the laser beam to an irradiance of $6.62 \mu \mathrm{W} / \mathrm{cm}^{2}$ at the cornea, keeping the cumulative exposure about one log unit below the maximum permissible exposure level (ANSI Z136.1, 1993). The neutral-density filter also prevented image saturation because it restricted the peak intensity of an individual aerial image to about two-thirds of the usable intensity range.

Pupil diameters of the 21 eyes ranged from 2.0 to $5.5 \mathrm{~mm}$ (Table 1), with an average diameter of $3.9 \mathrm{~mm}$. Pupil diameters increased with age (linear regression analysis: pupil diameter in $\mathrm{mm}=1.29+0.58 \times$ age in weeks; $\left.\mathrm{R}^{2}=0.53, \mathrm{p}=0.0002\right)$. The untreated birds were older than the experimental birds and, hence, had larger pupils; the average pupil diameter of the untreated birds was $4.5 \mathrm{~mm}$. The variability in pupil diameters is large for chicks of this age group, and this may be due to incomplete cycloplegia in the birds treated with vecuronium bromide. The CNS eyes tended to have larger pupils than cyclopleged eyes of the same age, further suggesting that the cycloplegia may have been incomplete. For all but three of the eyes, the pupil diameter was $<5 \mathrm{~mm}$; thus for the majority of eyes, both the incoming beam and the beam reflected out of the eye were limited by the eye's pupil diameter, producing a symmetrical double-pass aerial image. ${ }^{17}$ The pupil diameter was slightly larger than $5 \mathrm{~mm}$ in three of the untreated chick eyes, so in these eyes, the reflected beam was slightly larger in diameter than the incoming beam. However, the difference in incoming and outgoing beams in these three cases is small and probably had little effect on the optical quality estimates. The full width at half-maximum (FWHM) data and the aerial images from these eyes do not appear to be different from images taken from the other eyes, so we included them in the data analysis.

\section{Image Analysis}

The CCD format used in recording the four 6-week-old untreated birds was a $512 \times 512$ array of $24-\mu \mathrm{m}$ square pixels ( 16 bits per pixel intensity scale) with an image resolution of $0.165 \mathrm{~min}$ arc per pixel, whereas the CCD format for the remaining birds was a $576 \times 384$ array of $22-\mu \mathrm{m}$ square pixels ( 12 bits per pixel intensity scale) with an image resolution of $0.152 \mathrm{~min}$ arc per pixel. Aerial images were analyzed using Matlab (Mathworks). A background noise image was first subtracted from each aerial image; the background noise included the noise of the cooled CCD camera and any effects of stray light in the optical system. The intensity peak was then located, and the aerial image was truncated to the central 
$256 \times 256$ pixels, corresponding to an angular area of approximately $40 \times 40 \mathrm{~min}$ arc. Spline interpolation was applied to the image to increase resolution, and the image intensity profile was averaged for all orientations. The FWHM of the image was defined as twice the width of the average radial profile at half its maximum intensity. For each eye, the three individual images that had the smallest FWHM values were averaged after background subtraction and registration of their intensity peaks; a FWHM value was subsequently determined for this averaged image. Image averaging minimized the noise associated with laser speckle. Fig. 2 shows an example of an averaged double-pass aerial image from an untreated eye, truncated to show the central $20 \times 20 \mathrm{~min}$ arc, and a plot of its radial intensity profile. The FWHM value of this image is 1.485 min arc. Single-pass MTF's were derived from these averaged double-pass aerial images by taking the square root of the aerial image's two-dimensional Fourier transform.

\section{RESULTS}

\section{Optical Properties of Untreated Chick Eyes}

Double-pass aerial images from chick eyes were similar in appearance to those for human eyes. Shown in Fig. 3 is a series of aerial images captured at different focus positions from a 6-weekold untreated chick eye with a 5-mm pupil. Each image is the average of three images taken at the same focus position and shows the central $19 \times 19 \mathrm{~min}$ arc of the averaged image. The dioptric value under each image indicates the power of the trial lens that had been placed in front of the eye during recording. The FWHM value of each averaged image is also shown in min arc. Because of variability between images captured at the same focus position, none of the averaged images in this series show perfect focus. Possible sources of variability include small fluctuations in eye alignment, tear film quality, and accommodation due to incomplete cycloplegia.

The single-pass MTF's derived from these six images are shown in Fig. 4a. For the high spatial frequencies, retinal image quality was best with the $+1.25 \mathrm{D}$ lens, but it was better for lower spatial frequencies with less-positive lenses $(-0.75$ and $+0.25 \mathrm{D})$. MTF's derived from a through-focus series on another untreated chick eye
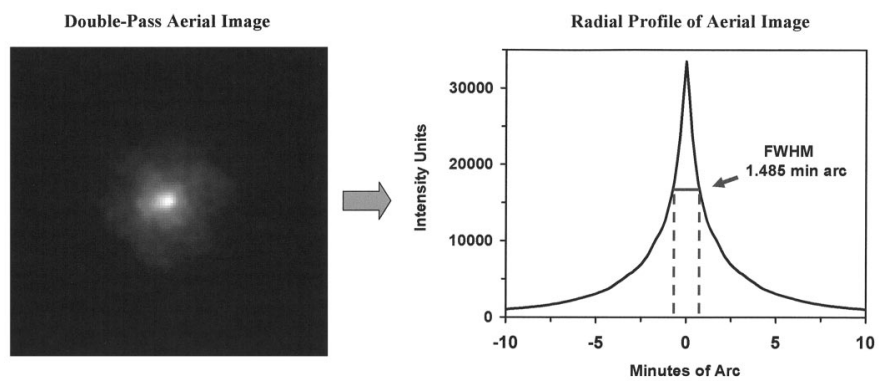

\section{FIGURE 2.}

Example of a double-pass aerial image (left) from an untreated chick eye with a pupil diameter of $5.0 \mathrm{~mm}$. The aerial image shown is the average of the three best individual images obtained on this eye. The image represents the central $19 \times 19 \mathrm{~min}$ arc of the original image. The radial profile of this image (right) in min arc is the average intensity profile for all orientations of the double-pass image. The left side (negative $x$ values) of the profile was obtained by reflecting the values from the right side. The full width at half-maximum (FWHM) is $1.485 \mathrm{~min}$ arc for this image. are shown in Fig. 4b. Here, also, the best image modulation for low spatial frequencies was obtained with a more negative lens $(-1.50$ D) than that producing the best modulation for high spatial frequencies $(-0.25 \mathrm{D})$. This pattern of results is consistent with positive spherical aberration, ${ }^{18,19}$ although we are uncertain whether all chick eyes would show this effect because we did not record through-focus series for all eyes.

The optimal retinal image quality of each eye was determined by averaging its three best individual aerial images, even if these three images were obtained with slightly different lens powers. This approach seems justifiable for eyes without complete through-focus data because for the two through-focus series shown in Fig. 4, the average lens power that produced the three best aerial images tended to agree with the lens power producing the best optical performance for each eye. For example, the three best individual aerial images for the eye in Fig. 4 a were obtained with an average lens power of $+0.33 \mathrm{D}$, which is consistent with the high overall MTF obtained with the $+0.25 \mathrm{D}$ lens. For the eye in Fig. $4 \mathrm{~b}$, the three best individual aerial images were obtained with an average lens power of $0.00 \mathrm{D}$ (plano), and the plano condition produced the highest image modulation across the entire spatial frequency range.

For the 10 eyes from untreated birds, the FWHM values corresponding to the average of each eye's three best images ranged from 1.16 to $2.08 \mathrm{~min}$ arc, with an overall average value of $1.60 \mathrm{~min}$ arc and a standard deviation of $\pm 0.29 \mathrm{~min}$ arc. MTF's computed for these same 10 untreated eyes and optimal focus conditions are shown in Fig. 5. Results for the 6-week-old birds (open circles) and 5-week-old birds (filled circles) are shown separately because of differences in their mean pupil diameters (4.8 and $3.25 \mathrm{~mm}$, respectively) and changes in the CCD camera format used in recording. Nonetheless, the MTF's representing these two sets of untreated eyes are very similar. The double-pass MTF's for the chicken are also compared with double-pass MTF's obtained on adult human eyes. The two dashed curves from Guirao et al. ${ }^{20}$ were obtained with 4- and 6-mm pupil diameters and 543-nm light. The solid line represents the human eye MTF for an 8-mm pupil curve, derived from the parametric model of Deeley et al. ${ }^{21}$ The chicken MTF's are lower than the human MTF's for similar pupil diameters.

\section{Effect of Refractive Error on Optical Quality}

Despite the small sample size and the varied nature of the subject group, refractive error apparently has an effect on the optical quality of the chicken eye. Table 2 summarizes the average FWHM values obtained when both the untreated and experimental birds were grouped by their refractive errors (not treatments). Eyes with refractive error magnitude of $<1.00 \mathrm{D}$ were classed as emmetropic. Lens-treated myopic eyes exhibited worse optical quality than hyperopic and emmetropic eyes. These differences were statistically significant in unpaired two-tailed t-tests (myopic vs. hyperopic eyes, $\mathrm{p}=0.007$; and myopic vs. emmetropic eyes, $\mathrm{p}=0.047$; the test against emmetropic eyes excluded the fellow control eyes of the myopic eyes). This trend can also be seen in Fig. 6, which shows FWHM values plotted as a function of refractive error. Interestingly, the untreated and fellow control eyes, which were approximately emmetropic, showed a broad spread of FWHM values. The 

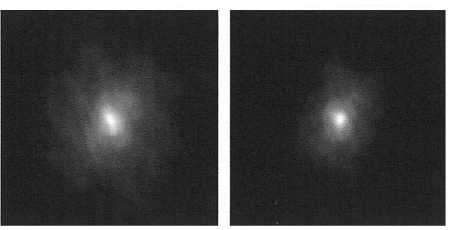

$-1.25 D$

$2.706 \mathrm{~min}$

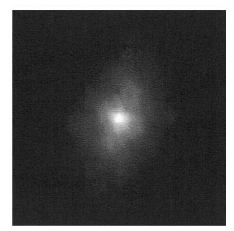

$-0.25 \mathrm{D}$

$1.815 \mathrm{~min}$

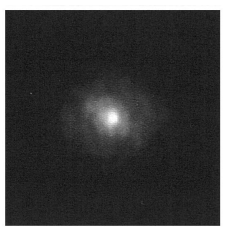

$+0.25 \mathrm{D}$

$2.079 \min$



$+0.75 \mathrm{D}$

$1.815 \mathrm{~min}$

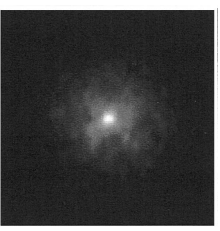

$+1.25 \mathrm{D}$

$1.782 \mathrm{~min}$

\section{FIGURE 3.}

Through-focus series of double-pass aerial images for an untreated chick eye (bird F, OD) with a pupil diameter of $5 \mathrm{~mm}$. Each image is the average of three single images taken at the same focus level. Lens power is the value of the trial lens in place during each measurement. Each image subtends $19 \times 19 \mathrm{~min}$ arc.
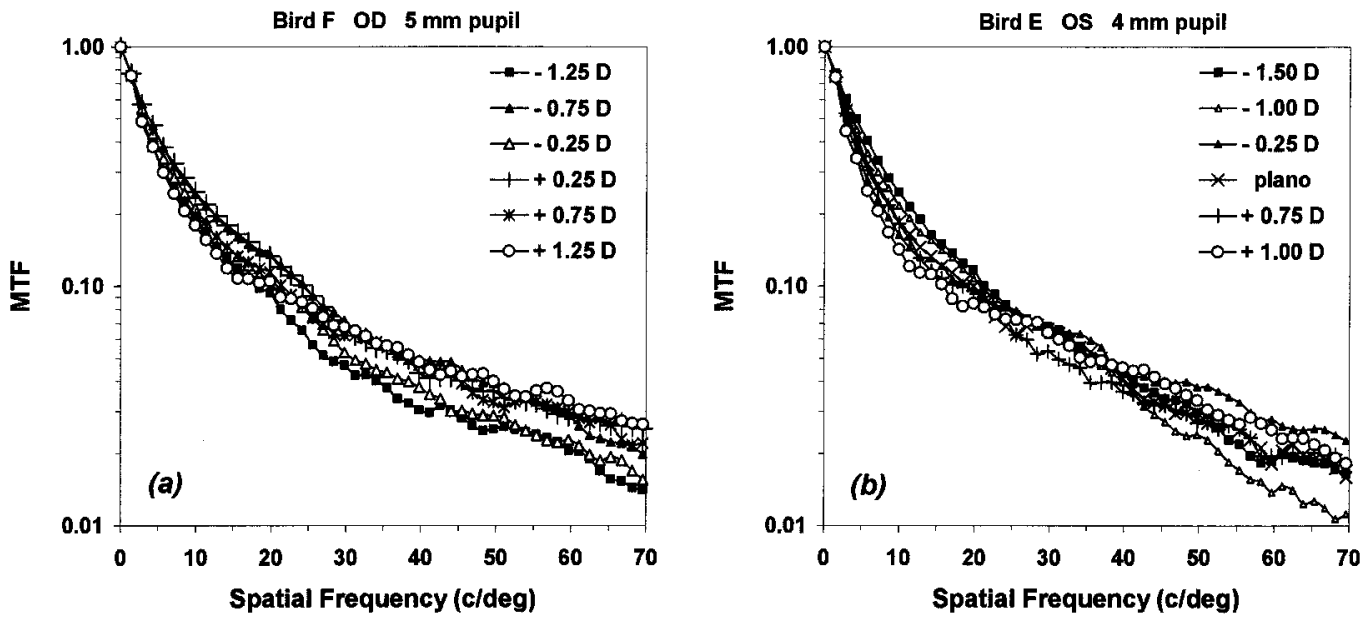

\section{FIGURE 4.}

a: Single-pass modulation transfer functions (MTF's) obtained from the through-focus series in Fig. 3. Each MTF is the square root of the Fourier transform of its corresponding double-pass aerial image. The MTF ratio represents the ratio of retinal image contrast to object contrast. Lens powers represent the trial lens power in place during measurements. b: Single-pass MTF's obtained from a through-focus series on another untreated eye (bird E, OS) with a 4-mm pupil.

regression line shown in Fig. 6 is fit to all the data, including the untreated and fellow control eyes. A regression line fit to the experimental eye data alone (filled symbols) is described by the following equation: FWHM $=-0.504 \times$ (Diopters) $+1.7732\left(\mathrm{R}^{2}\right.$ $=0.71 ; \mathrm{p}=0.008)$. These trends suggest that hyperopic eyes have better optical quality than emmetropic and myopic eyes. However, this apparent difference between hyperopic and emmetropic eyes (excluding fellow control eyes of the hyperopic eyes) (Table 2) was not significantly different ( $\mathrm{p}=0.099$, unpaired two-tailed $\mathrm{t}$-test), presumably reflecting the small number of hyperopic eyes and the large scatter in the data from emmetropic eyes.

\section{DISCUSSION}

\section{Optical Quality in the Chicken Eye and Its Relation to Spatial Acuity}

Optical modulation transfer functions that exceed the sampling capacity of the retina can lead to spatial aliasing and are thus undesirable. In the case of the human eye, its optical quality is matched to the human eye's foveal cone density, allowing high contrast acuity up to about 45 to 50 cpd but preventing aliasing that would occur at frequencies above about $60 \mathrm{cpd}^{22,23}$ Specifically, the human single-pass retinal PSF width for a $4-\mathrm{mm}$ pupil is estimated to be $0.8 \mathrm{~min}$ arc, ${ }^{24}$ which is about 1.5 times the centerto-center spacing of central foveal cones. ${ }^{22}$ On the other hand, at $20^{\circ}$ retinal eccentricity, the single-pass retinal PSF width is only slightly larger $\left(1 \mathrm{~min} \operatorname{arc}^{24}\right)$, whereas the neural sampling, in this case determined by the center-to-center spacing of the midget ganglion cells, is much coarser (about $7 \mathrm{~min}$ arc). ${ }^{25}$ The aliasing that can be experienced at peripheral retinal locations reflects the fact that the optical quality of the human eye is better than that required by the peripheral neural grain.

Behavioral measures of spatial acuity in the chicken range from $1.5 \mathrm{cpd}^{26}$ for 1 - to 25 -day-old chicks to about $7 \mathrm{cpd}$ for 6-monthold birds. ${ }^{27} \mathrm{~A}$ more recent estimate based on an optokinetic nystagmus paradigm is slightly higher, between 7.7 and $8.6 \mathrm{cpd}$ for 8 -day-old birds. ${ }^{28}$ The latter value is closer to the figures of 8.1 and $13 \mathrm{cpd}$, which represent Nyquist limits calculated from ganglion cell density estimates for the chick central retina. ${ }^{29,30}$ The latter approach has been widely applied in estimating spatial acuity for a range of animals ${ }^{31-33}$ and rests on the assumption that there is considerable convergence of cone signals onto retinal ganglion cells. In the central retina of a 3- to 4-week-old chick, the peak ganglion cell density is about 30,000 cells $/ \mathrm{mm}^{2}$, and the minimum center-to-center ganglion cell spacing about $5.8 \mu \mathrm{m} .{ }^{30}$ 


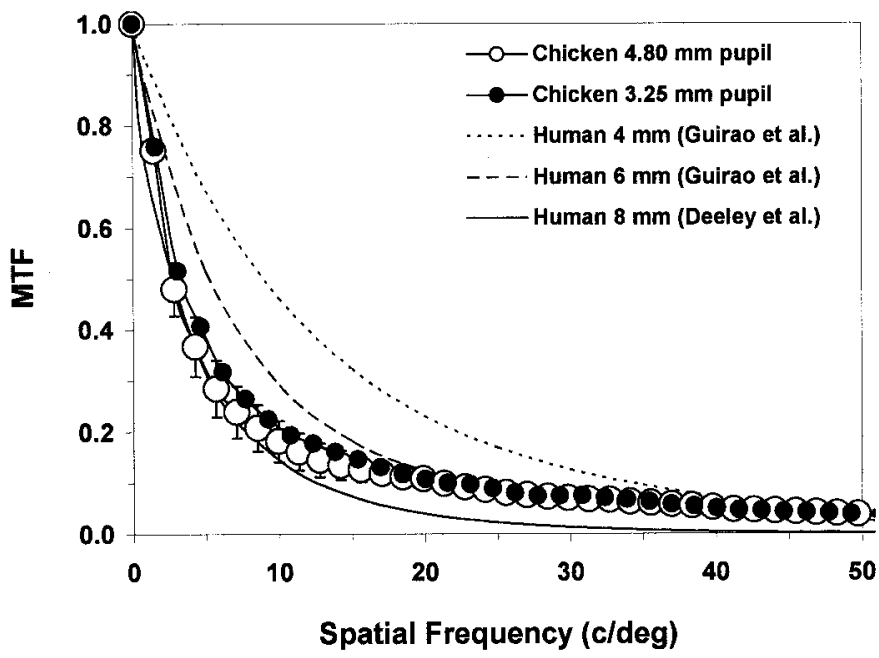

FIGURE 5.

Single-pass modulation transfer functions (MTF's) obtained on the untreated chick eyes. Open circles represent the average MTF obtained from the best images on the eight 6-week-old untreated eyes; the average pupil diameter was $4.80 \mathrm{~mm}$. Filled circles represent the average MTF obtained from the best images of the two 5-week-old untreated eyes, with an average pupil diameter of $3.25 \mathrm{~mm}$. Error bars on data points are the SD of the average MTF's. Dotted and dashed lines are human MTF's for 4- and 6-mm pupils, respectively, obtained with the double-pass technique and 543-nm light. ${ }^{20}$ The solid line is the human MTF calculated for an 8-mm pupil using the model proposed by Deeley et al. ${ }^{21}$

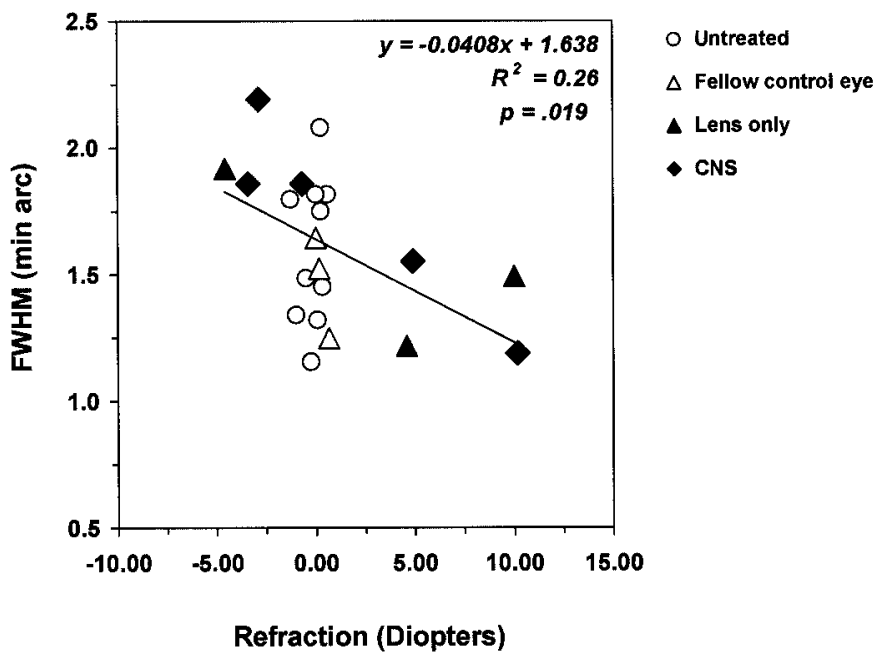

\section{FIGURE 6.}

Double-pass aerial image width (full width at half-maximum [FWHM]), averaged from the three best images from each eye, as a function of the refraction of the eye in diopters. Refraction values were obtained as the average power of the trial lenses in place during recordings of the three best individual images. Open circles are data for the 10 untreated eyes (birds A to $\mathrm{F}$ in Table 1); open triangles are data for the fellow control eyes of the monocular lens treatment birds (G to I, left eyes); solid triangles are data for the lens-treated eyes of the monocular lens treatment birds ( $\mathrm{G}$ to $\mathrm{I}$, right eyes); solid diamonds are data for the five birds (J to $\mathrm{N}$ ) that underwent ciliary nerve section (CNS), including the four "lens + CNS" birds.

How well does the optical quality of the chick eye match the neural grain of the retina? The single-pass retinal PSF width is likely to be smaller than the $1.60 \mathrm{~min}$ arc double-pass aerial image
TABLE 2.

Summary of the optical quality of the chicken eyes assessed in this study, grouped by their refractive error outcomes

\begin{tabular}{|c|c|c|}
\hline Refractive Error Type & $\begin{array}{l}\text { Refractive Error } \\
\text { Range (D) }\end{array}$ & $\begin{array}{c}\mathrm{FWHM} \pm \mathrm{SD}^{a} \\
\quad(\min \mathrm{arc})\end{array}$ \\
\hline $\begin{array}{l}\text { Lens-treated hyperopic } \\
\text { eyes }^{b}(\mathrm{~N}=4)\end{array}$ & +4.58 to +10.17 & $1.362 \pm 0.187$ \\
\hline $\begin{array}{l}\text { Emmetropic eyes }{ }^{c} \\
\quad(\mathrm{~N}=12)\end{array}$ & -0.50 to +0.67 & $1.595 \pm 0.279$ \\
\hline $\begin{array}{l}\text { Untreated myopic } \\
\text { eyes }(N=2)\end{array}$ & -1.00 to -1.29 & $1.568 \pm 0.323$ \\
\hline $\begin{array}{l}\text { Lens-treated myopic } \\
\text { eyes }^{b}(\mathrm{~N}=3)\end{array}$ & -2.87 to -4.58 & $1.989 \pm 0.178$ \\
\hline
\end{tabular}

recorded in this study. Single-pass PSF's only slightly narrower than the latter value, between 1 to $1.5 \mathrm{~min}$ arc, should cover about 2.2 to $3.3 \mu \mathrm{m}$ on the retina or about one-half of the minimum ganglion cell center-to-center spacing. This would imply that for the chick eye, optical quality exceeds the neural grain, even in the high-density central retina, by an amount analogous to the situation in the parafovea or midperiphery of the human eye. However, the FWHM estimates reported here were determined from optimally focused monochromatic images and do not include the effects of chromatic aberration. Under white light conditions, the chick eye exhibits over $3 \mathrm{D}$ of chromatic aberration. ${ }^{34} \mathrm{~A}$ recent study $^{35}$ of the human eye has noted that monochromatic aberrations can partially compensate for the effects of longitudinal chromatic aberration. Even with this compensation, however, the polychromatic MTF is lower than the monochromatic MTF. We are uncertain whether the monochromatic aberrations in the chick eye would also tend to compensate chromatic aberration. Thus, it is likely that the functional optical quality of the chick eye is poorer than the monochromatic FWHM numbers reflect and more closely matched to the neural grain of the central retina.

\section{Refractive Error and Optical Quality}

One question of interest in the context of myopia research is whether myopic eyes have higher than normal optical aberrations. Retinal image degradation, for example produced experimentally with diffusing goggles, results in myopia and, thus, it is plausible that higher than normal aberrations might also produce myopic changes. Our data indicate that myopic eyes have poorer optical quality than hyperopic eyes, even though the average amount of myopia was only modest (average of $-3.62 \mathrm{D}$ in the experimental eyes). That these refractive errors were experimentally induced argues for the differences in optical quality being the result of, rather than the cause of, the induced refractive changes.

The apparent link between myopia and reduced optical quality described here for the chick eye is consistent with evidence from human-based studies. Specifically in human eyes, wavefront aberrations of the cornea ${ }^{2}$ and whole eye $e^{3,36}$ increase with the degree of 
myopia, and compared with emmetropic eyes, myopic eyes are likely to show higher monochromatic aberrations. ${ }^{1}$ Whether the increased aberrations reported for myopic human eyes is a cause or an effect of the refractive error remains to be resolved. There has been less study of aberrations in hyperopic human eyes, but, in hyperopia up to $+4.50 \mathrm{D}$, whole-eye aberrations do not appear to differ from those of emmetropic or low myopic eyes. ${ }^{37}$

The origin of the altered aberrations was not investigated in the current study. However in the human eye, the spherical aberrations of cornea and crystalline lens tend to neutralize each other, with the lens manifesting negative spherical aberration and the cornea manifesting positive spherical aberration. ${ }^{38}$ This balance of corneal and internal spherical aberrations appears to be preserved even with high amounts of myopia where an increase in positive corneal spherical aberration is compensated by an increase of negative internal spherical aberration. ${ }^{3}$ Thus, the increase in whole-eye aberrations in human myopia appears to be due to increased amounts of third-order asymmetric (coma-like) aberrations. ${ }^{3}$ This suggests that myopic eyes may have greater misalignment or tilting of optical surfaces than emmetropic eyes. Whether this pattern of results also explains the increased aberrations in myopic chick eyes is uncertain. In the developing chick eye, crystalline lenses excised from treated hyperopic eyes and myopic eyes have reduced optical quality compared with their fellow control eyes. ${ }^{39}$ However, this effect of myopia on aberrations is not apparent when lenses are measured in situ. ${ }^{40}$

Monochromatic aberrations have also been considered from another perspective in the context of emmetropization as a possible cue to defocus that could allow eyes to distinguish myopic from hyperopic defocus. ${ }^{8}, 9$ The MTF examples shown in Fig. 4 indicate that aberrations in the chick eye could serve this role. Specifically, the curves representing the two extremes of the defocus range switch over at some intermediate frequency, and, thus by implication, retinal image contrast will vary in opposite directions at high and low spatial frequencies, depending on the sign of defocus. Thus, a visual mechanism that compares the contrast of high to low spatial frequencies theoretically could differentiate the sign of defocus. Nonetheless, monochromatic aberrations are unlikely to be the only defocus cues used during emmetropization because this process operates over a wide range of pupil diameters and with relatively poor retinal images in the developing chick eye. ${ }^{41}$

In conclusion, the optical quality of the developing chick eye appears to be suitably matched with its retinal resolution capacity after chromatic aberration is also taken into account. In the case of experimentally induced refractive errors, myopic eyes had poorer optical quality than emmetropic or hyperopic eyes, consistent with a growth-mediated rather than a causal origin to these differences.

\section{ACKNOWLEDGMENTS}

We thank Dr. Debora Nickla of the New England College of Optometry for her technical assistance and Dr. Stephen Burns of the Schepens Eye Research Institute for his advice and the loan of equipment.

Support for this research was provided by National Eye Institute, National Institutes of Health grants EY04395 to S. Burns, EY11228 to D. Troilo, EY12392 to C. Wildsoet, and EY12847 to N. Coletta.

S. Marcos was supported by the Human Frontier Science Program LTI 0542/1997-B and Fulbright 163/2000.
This research was presented as a paper 42 at the annual meeting of the Association for Research in Vision and Ophthalmology on May 4, 2000, in Ft. Lauderdale, FL.

Received May 23, 2002; revision received September 30, 2002.

\section{REFERENCES}

1. Collins MJ, Wildsoet CF, Atchison DA. Monochromatic aberrations and myopia. Vision Res 1995;35:1157-63.

2. He JC, Gwiazda J, Held R, Thorn F. Wave-front aberrations in the cornea and the whole eye for emmetropes and myopes. Invest Ophthalmol Vis Sci 2001;42:S99.

3. Marcos S, Barbero S, Llorente L. The sources of optical aberrations in myopic eyes. Invest Ophthalmol Vis Sci 2002;43:S1510.

4. Bartmann M, Schaeffel F. A simple mechanism for emmetropization without cues from accommodation or colour. Vision Res 1994;34: 873-6.

5. Hung LF, Wallman J, Smith EL III. Vision-dependent changes in the choroidal thickness of macaque monkeys. Invest Ophthalmol Vis Sci 2000;41:1259-69.

6. Schaeffel F, Howland HC. Visual optics in normal and ametropic chickens. Clin Vision Sci 1988;3:83-98.

7. Irving EL, Sivak JG, Curry TA, Callender MG. Chick eye optics: zero to fourteen days. J Comp Physiol (A) 1996;179:185-94.

8. Campbell MC, Priest D, Hunter JJ. The importance of monochromatic aberrations to detecting defocus in retinal images. Invest Ophthalmol Vis Sci 2001;42:S98.

9. Wilson BJ, Decker KE, Roorda A. Monochromatic aberrations provide an odd-error cue to focus direction. J Opt Soc Am (A) 2002;19: 833-9.

10. Bonds AB. Optical quality of the living cat eye. J Physiol 1974;243: 777-95.

11. Artal P, Herreros de Tejada P, Munoz Tedo C, Green DG. Retinal image quality in the rodent eye. Vis Neurosci 1998;15:597-605.

12. Campbell FW, Gubisch RW. Optical quality of the human eye. J Physiol (Lond) 1966;186:558-78.

13. Santamaria J, Artal P, Bescos J. Determination of the point-spread function of human eyes using a hybrid optical-digital method. J Opt Soc Am (A) 1987;4:1109-14.

14. Schmid KL, Wildsoet CF. Effects on the compensatory responses to positive and negative lenses of intermittent lens wear and ciliary nerve section in chicks. Vision Res 1996;36:1023-36.

15. Nickla DL, Wildsoet C, Wallman J. Visual influences on diurnal rhythms in ocular length and choroidal thickness in chick eyes. Exp Eye Res 1998;66:163-81.

16. Artal P, Marcos S, Navarro R, Williams DR. Odd aberrations and double-pass measurements of retinal image quality. J Opt Soc Am (A) 1995;12:195-201.

17. Artal P, Iglesias I, Lopez-Gil N, Green DG. Double-pass measurements of the retinal-image quality with unequal entrance and exit pupil sizes and the reversibility of the eye's optical system. J Opt Soc Am (A) 1995;12:2358-66.

18. Green DG, Campbell FW. Effect of focus on the visual response to a sinusoidally modulated spatial stimulus. J Opt Soc Am 1965;55: 1154-7.

19. Charman WN, Jennings JA. The optical quality of the monochromatic retinal image as a function of focus. Br J Physiol Opt 1976;31: 119-34.

20. Guirao A, Gonzalez C, Redondo M, Geraghty E, Norrby S, Artal P. Average optical performance of the human eye as a function of age in a normal population. Invest Ophthalmol Vis Sci 1999;40:203-13.

21. Deeley RJ, Drasdo N, Charman WN. A simple parametric model of 
the human ocular modulation transfer function. Ophthalmic Physiol Opt 1991;11:91-3.

22. Williams DR. Visibility of interference fringes near the resolution limit. J Opt Soc Am (A) 1985;2:1087-93.

23. Thibos LN, Bradley A, Zhang X. Effect of ocular chromatic aberration on monocular visual performance. Optom Vis Sci 1991;68: 599-607.

24. Navarro R, Artal P, Williams DR. Modulation transfer of the human eye as a function of retinal eccentricity. J Opt Soc Am (A) 1993;10: 201-12.

25. Curcio CA, Allen KA. Topography of ganglion cells in human retina. J Comp Neurol 1990;300:5-25.

26. Over R, Moore D. Spatial acuity of the chicken. Brain Res 1981;211: 424-6.

27. Johnson HM. Visual pattern-discrimination in the vertebrate: II. Comparative visual acuity in the dog, the monkey and the chick. J Animal Behav 1914;4:340-61.

28. Schmid KL, Wildsoet CF. Assessment of visual acuity and contrast sensitivity in the chick using an optokinetic nystagmus paradigm. Vision Res 1998;38:2629-34.

29. Troilo D, Xiong M, Crowley JC, Finlay BL. Factors controlling the dendritic arborization of retinal ganglion cells. Vis Neurosci 1996;13: 721-33.

30. Ehrlich D. Regional specialization of the chick retina as revealed by the size and density of neurons in the ganglion cell layer. J Comp Neurol 1981;195:643-57.

31. Snyder AW, Miller WH. Photoreceptor diameter and spacing for highest resolving power. J Opt Soc Am 1977;67:696-8.

32. Pettigrew JD, Dreher B, Hopkins CS, McCall MJ, Brown M. Peak density and distribution of ganglion cells in the retinae of microchiropteran bats: implications for visual acuity. Brain Behav Evol 1988; 32:39-56.

33. Schmid KL, Schmid LM, Wildsoet CF, Pettigrew JD. Retinal topog- raphy in the koala (Phascolarctos cinereus). Brain Behav Evol 1992;39: 8-16.

34. Schmid KL, Wildsoet CF. Contrast and spatial-frequency requirements for emmetropization in chicks. Vision Res 1997;37:2011-21.

35. McLellan JS, Marcos S, Prieto PM, Burns SA. Imperfect optics may be the eye's defence against chromatic blur. Nature 2002;417:174-6.

36. Bueno JM, Priest D, Campbell MCW. Optical aberrations of the eye as a function of refractive error in young subjects (Abstract WO4). OSA Annual Meeting Conference Program, 2000. Washington, DC: Optical Society of America, 2000:100.

37. Llorente L, Marcos S, Barbero S, Mareyo-Lloves J. How total and corneal aberrations change with standard LASIK surgery for hyperopia. Invest Ophthalmol Vis Sci 2002;43:S2066.

38. Artal P, Guirao A, Berrio E, Williams DR. Compensation of corneal aberrations by internal optics in the human eye. J Vis 2001;1:1-8. Available at: http://journalofvision.org/1/1/1. Accessed October 7, 2002.

39. Priolo S, Sivak JG, Kuszak JR, Irving EL. Effects of experimentally induced ametropia on the morphology and optical quality of the avian crystalline lens. Invest Ophthalmol Vis Sci 2000;41:3516-22.

40. Choh V, Sivak JG. Optical changes of accommodating lenses in myopic chicks. In: Thorn F, Troilo D, Gwiazda J, eds. Myopia 2000: Proceedings of the VIII International Conference on Myopia. Boston: New England College of Optometry Press, 2000:279-82.

41. Schaeffel F, Diether S. The growing eye: an autofocus system that works on very poor images. Vision Res 1999;39:1585-9.

42. Coletta NJ, Marcos S, Wildsoet C, Troilo D, Burns SA. Optical quality of the chicken eye. Invest Ophthalmol Vis Sci 2000;41:S738.

Nancy J. Coletta

New England College of Optometry

424 Beacon Street

Boston, MA 02115

e-mail: colettan@ne-optometry.edu 